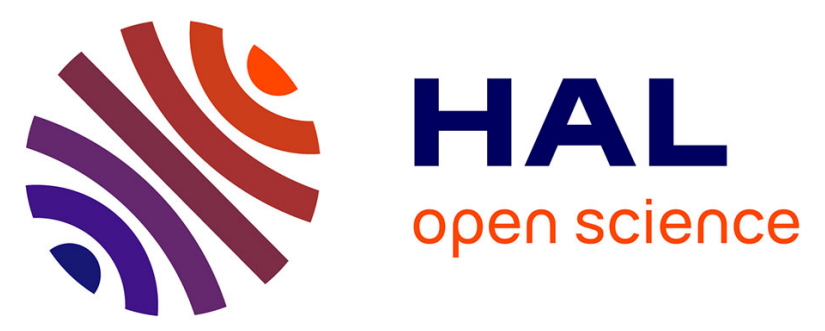

\title{
Macrophage migrating inhibitory factor expression is associated with Trypanosoma brucei gambiense infection and is controlled by trans-acting expression quantitative trait loci in the Guinean population
}

\author{
Justin Windingoudi Kaboré, Oumou Camara, Hamidou Ilboudo, Paul \\ Capewell, Caroline Clucas, Anneli Cooper, Jacques Kaboré, Mamadou \\ Camara, Vincent Jamonneau, Christiane Hertz-Fowler, et al.
}

\section{- To cite this version:}

Justin Windingoudi Kaboré, Oumou Camara, Hamidou Ilboudo, Paul Capewell, Caroline Clucas, et al.. Macrophage migrating inhibitory factor expression is associated with Trypanosoma brucei gambiense infection and is controlled by trans-acting expression quantitative trait loci in the Guinean population. Infection, Genetics and Evolution, 2019, 71, pp.108 - 115. 10.1016/j.meegid.2019.03.021 . hal-03485640

\author{
HAL Id: hal-03485640 \\ https://hal.science/hal-03485640
}

Submitted on 20 Dec 2021

HAL is a multi-disciplinary open access archive for the deposit and dissemination of scientific research documents, whether they are published or not. The documents may come from teaching and research institutions in France or abroad, or from public or private research centers.
L'archive ouverte pluridisciplinaire HAL, est destinée au dépôt et à la diffusion de documents scientifiques de niveau recherche, publiés ou non, émanant des établissements d'enseignement et de recherche français ou étrangers, des laboratoires publics ou privés.

\section{다)(1) $(5$}

Distributed under a Creative Commons Attribution - NonCommerciall 4.0 International 
1 Macrophage migrating inhibitory factor expression is associated with Trypanosoma brucei gambiense infection and is controlled by trans-acting expression quantitative trait 3 loci in the Guinean population

4 Justin Windingoudi Kaboré ${ }^{1}$, Oumou Camara ${ }^{2}$, Hamidou Ilboudo ${ }^{3}$, Paul Capewell ${ }^{4}$, Caroline 5 Clucas $^{4}$, Anneli Cooper ${ }^{4}$, Jacques Kaboré ${ }^{1,5}$, Mamadou Camara ${ }^{2}$, Vincent Jamonneau ${ }^{6}$, 6 Christiane Hertz-Fowler ${ }^{7}$, Adrien Marie Gaston Bélem ${ }^{5}$, Enock Matovu ${ }^{8}$, Annette Macleod ${ }^{4}$, 7 Issa Sidibé ${ }^{1}$, Harry Noyes ${ }^{9 \mathbb{I}}$ and Bruno Bucheton ${ }^{2,6 * \pi}$, For the TrypanoGEN Research Group, as 8 member of the H3Africa Consortium.

$9{ }^{1}$ Centre International de Recherche-Développement sur l'Élevage en zone Subhumide 10 (CIRDES), Unité des Maladies à Vecteurs et Biodiversités (UMaVeB), Bobo-Dioulasso, Burkina Faso; ${ }^{2}$ Ministère de la Santé et de l'Hygiène Publique, Programme National de Lutte contre la Trypanosomiase Humaine Africaine (PNLTHA), Conakry, Guinée; ${ }^{3}$ Institut de Recherche en Sciences de la Santé (IRSS), Unité de Recherche Clinique de Nanoro (URCN), Parasitology, Glasgow, United Kingdom; ${ }^{5}$ Université Nazi Boni (UNB), Bobo-Dioulasso,

Research, Liverpool, United Kingdom.

23 E-mail: bruno.bucheton@ird.fr

24 II These authors contributed equally to this work. 


\section{Abstract}

Infection by Trypanosoma brucei gambiense is characterized by a wide array of clinical outcomes, ranging from asymptomatic to acute disease and even spontaneous cure. In this study, we investigated the association between macrophage migrating inhibitory factor $(M I F)$, an important pro-inflammatory cytokine that plays a central role in both innate and acquired immunity, and disease outcome during T. b. gambiense infection. A comparative expression analysis of patients, individuals with latent infection and controls found that MIF had significantly higher expression in patients $(n=141 ; 1.25 \pm 0.07 ; p<0.0001)$ and latent infections $(n=25 ; 1.23 \pm 0.13 ; p=0.0005)$ relative to controls $(n=46 ; 0.94 \pm 0.11)$. Furthermore, expression decreased significantly after treatment (patients before treatment $n=33 ; 1.40 \pm 0.18$ versus patients after treatment $n=33 ; 0.99 \pm 0.10, p=0.0001)$. We conducted a genome wide eQTL analysis on 29 controls, 128 cases and 15 latently infected individuals for whom expression and genotype data were both available. Four loci, including one containing the chemokine CXCL13, were found to associate with MIF expression. Genes at these loci are candidate regulators of increased expression of $M I F$ after infection. Our study is the first data demonstrating that $M I F$ expression is elevated in T. b. gambiense-infected human hosts but does not appear to contribute to pathology.

\section{Introduction}

Found in sub-Saharan countries, human African trypanosomiasis (HAT), or sleeping sickness, is a neglected tropical disease. It is caused by Trypanosoma brucei gambiense in western and central Africa, and T. $b$. rhodesiense in eastern Africa. The two forms of the disease are considered lethal without treatment and both are transmitted by tsetse flies of the genus Glossina (order Diptera) (Buscher et al., 2017; Simarro et al., 2010). T. b. gambiense causes 97\% of all HAT cases and is classically described as a chronic disease with an early haemolymphatic phase (stage 1) characterized by non-specific signs. This is followed by a meningoencephalitic phase (stage 2) in which the parasites penetrate the blood brain barrier, leading to neurological disorders and more specific signs characterised by sleep disorder from which the name sleeping sickness is derived, consists of daytime somnolence and sudden overwhelming sleep urges, and nocturnal insomnia (Buscher et al., 2017; Checchi et al., 2008). However, findings show that $T$. $b$. gambiense infection can lead to a wide array of clinical outcomes, ranging from acute disease that progresses rapidly to the second stage (Garcia et al., 2000; Truc et al., 1997) to spontaneous self-cures (Checchi et al., 2008; Jamonneau et al., 2012) 
and even latent infections with very low blood parasitaemia that, in most cases, are not detectable by microscopy. Long-term follow up has shown that this latter type of latent infection may persist for several years (Jamonneau et al., 2012).

The pathogenesis of T. $b$. gambiense HAT is poorly understood as disease development is dependent on the host immune response, the genetic variability of the parasites and the hostparasite interactions (Garcia et al., 2006). To address this, a candidate gene study was recently performed in Guinea to explore the association between genes involved in host immunity and the disease. This study found significant associations in the APOL1 gene a component on the trypanosome lytic factor (TLF) that confers resistance to T. b. brucei infection and also with rs1818879 of interleukin-6 (IL6) (Cooper et al., 2017; Kabore et al., 2017; Vanhamme et al., 2003). Several suggestive associations were observed at the macrophage migration inhibitory factor $(M I F)$ gene locus, although these did not remain significant after Bonferroni correction (Kabore et al., 2017). Although we did not observe significant genetic association at the MIF locus in our Guinean HAT cohort, MIF is nevertheless known as an important pro-inflammatory cytokine that plays a central role in the control of both innate and acquired immunity in a number of diseases (Calandra, 2003; Renner et al., 2005). Elevated serum levels of MIF have been found in patients with malaria (McDevitt et al., 2006), viral hepatitis (Kimura et al., 2006), HIV infection (Regis et al., 2010), rheumatoid arthritis (Morand et al., 2002), sepsis (Bozza et al., 2004), vasculopathy (Zernecke et al., 2008), and Chagas disease (Cutrullis et al., 2013). Importantly, it was also shown that MIF mediates the pathogenic inflammatory immune response and increases the recruitment of inflammatory monocytes and neutrophils promoting the most prominent pathological features of experimental trypanosome infections (Stijlemans et al., 2014). Regarding Trypanosoma cruzi, MIF was also shown to play an important role in the host defence against acute infections, favouring the production of the pro-inflammatory cytokines during the early phase of infection (Reyes et al., 2006). These studies have prompted us to evaluate MIF expression in human T. b. gambiense infections.

To establish a potential role for MIF in determining T. $b$. gambiense clinical diversity, we examined $M I F$ transcript expression in a Guinean cohort. For this study, $M I F$ mRNA expression levels were determined by qPCR in HAT patients before and after treatment, individuals harbouring long term latent infections and endemic uninfected controls. In addition, the availability of genome wide SNP data generated within the framework of the TrypanoGEN network for some of these individuals (Ilboudo et al., 2017) made it possible to identify expression quantitative trait loci (eQTLs) potentially involved in the regulation of $M I F$ 
expression. The identification of these loci contributes to our understanding of how MIF expression is controlled and may have relevance to a wide range of infectious and immunepathophysiological disease.

\section{Materials and methods}

\subsection{Ethics statement}

Samples were collected during medical surveys conducted by the national control program (NCP) of the Republic of Guinea according to the national HAT diagnostic procedures approved by the Ministry of Health in Guinea. All participants were informed of the objective of the study in their own language and signed an informed consent form. For participants less than 18 years of age, informed consent was obtained from their parents. Approval for this study was obtained from the «comité consultative de déontologie et d'éthique » (CCDE) of the «Institut de Recherche pour le Développement» (1-22/04/2013). This study is part of the TrypanoGEN project that aims to understand the genetic basis of human susceptibility to trypanosomiasis. Samples were archived in the TrypanoGen Biobank at CIRDES (Ilboudo et al., 2017).

\subsection{Study population}

All individuals participating in this study were identified during medical surveys performed between 2007 and 2011 by the NCP of the Republic of Guinea. Identification and sampling of study participants were performed in three active HAT foci (Forecariah, Dubreka and Boffa) located along the Guinean coast according to standard procedures describe previously (Ilboudo et al., 2011). For each participant, $5 \mathrm{ml}$ of blood was taken in anticoagulant blood collection tubes to collect plasma samples and buffy coats for further DNA extraction. Two additional ml of blood were also taken with PAXgene Blood RNA tubes (PreAnalytiX). All samples were frozen in a portable freezer at $-20^{\circ} \mathrm{C}$ in the field and were stored in the lab at $-80^{\circ} \mathrm{C}$. For all participants, the Card Agglutination Test for Trypanosomiasis (CATT) was performed. Direct microscopic examination of the buffy coat and/or the lymph node aspirate (if swollen lymph nodes were present) were performed if an individual was CATT positive, (Camara et al., 2010; Magnus et al., 1978). The immune trypanolysis test was performed at CIRDES on all plasma samples, as previously described (Jamonneau et al., 2010). For this study, 212 individuals were selected and three categories of phenotype were defined according to the following criteria: 
i. Cases or HAT patients $(n=141)$ : Parasitologically confirmed patients, with CATT plasma dilution end titers $\geq 1 / 4$ and trypanolysis test (TL) positive. All HAT patients diagnosed within this study were treated according to the NCP procedures. Patients with HAT stage 1 disease (CSF white cell counts, 5) were treated by daily injection of pentamidine for 8 consecutive days. Early stage 2 patients (CSF white cell counts 6-20) were treated with one injection of pentamidine every 2 days for 20 days (10 total injections). Late stage 2 patients (CSF white cell count, > 20) were treated with three cycles of melarsoprol injections (one-third of the dose on the $1^{\text {st }}$ day, two-thirds of the dose on the $2^{\text {nd }}$ day and a full dose on days 3 and 4 ) administered 10 days apart. Of the 141 patients, 33 were sampled again 6 months after treatment at their first follow-up and were included in this study to examine $M I F$ expression before and after treatment.

ii. Latent infections or Seropositive ( $n=25)$ : individuals with CATT plasma dilution end titers $\geq 1 / 4$, TL positive, parasitology negative (no trypanosomes detected by mAECT and/or by examination of cervical lymph node aspirate when cervical adenopathies were present) and who maintained this phenotype for least two years.

iii. Endemic controls ( $n=46)$ : Individuals with negative CATT results and negative TL, living in the same villages as a HAT patient and/or a seropositive subject.

The age and gender characteristics of the different study groups is given in supplementary Table S1.

\section{3. mRNA preparation and cDNA synthesis}

Total mRNA from blood was extracted with the PAXgene Blood RNA kit (PreAnalytiX) and quantified with a NanoDrop spectrophotometer (NanoDrop Technologies). The mRNA quality was checked using the RNA Integrity Number (RIN) on an Agilent 2100 Bioanalyzer with the RNA 600 Nano LabChip (Agilent Technologies). Total mRNA was reverse transcribed according to the manufacturer's instructions, using High Capacity cDNA Reverse Transcription kit, Applied Biosystems and RNase inhibitor (Applied Biosystems) at a final concentration of 1.0 U/ml. The cDNAs were stored at $-20^{\circ} \mathrm{C}$ and diluted to $1: 5$ with RNase-free water for use as a template in the real-time PCR analysis.

\subsection{MIF expression assays by RT-qPCR}

The real-time quantitative PCR analysis was conducted using the AriaMx machine (Agilent Technologies). All of the TaqMan RT-qPCR reagents, including the primers and probes, were 
151

152

153

154 155

purchased from Applied Biosystems. The RT-qPCR analysis was conducted using predesigned and optimized Assays on Demand (Applied Biosystems). The following assays were used: MIF (ID: Hs00236988_g1) and TBP (ID: Hs00427621_m1). The reaction parameters were a 2min at $50^{\circ} \mathrm{C}$ hold and a $5 \mathrm{~min}$ at $95^{\circ} \mathrm{C}$ hold, followed by 45 cycles of $15 \mathrm{~s}$ at $95^{\circ} \mathrm{C}$ for a melting, and $1 \mathrm{~min}$ at $60^{\circ} \mathrm{C}$ for annealing and extension. All of the measurements were performed in triplicate. A relative quantitation was conducted using TBP as a reference gene (Ledderose et al., 2011). The parameter cycle threshold $\left(\mathrm{C}_{\mathrm{t}}\right)$ was defined as the cycle number at which the fluorescence intensity exceeds a fixed threshold. Relative amounts of mRNA for target genes were calculated using the comparative $C_{t}$ method $\left(2^{-\Delta \Delta C t}\right)$. As the assays were optimized for PCR efficiency by the manufacturer, the mRNA-expression levels were estimated according to the delta-Ct values.

\subsection{Genotype data}

DNA was extracted from buffy coat (BC) samples using a Qiagen DNA extraction kit (QIAamp DNA Blood Midi Kit) following the manufacturer's instructions. DNA extracts were stored at $-20^{\circ} \mathrm{C}$. All DNA samples were quantified on a NanoDrop spectro-photometer, and shipped to Illumina, San Diego, California where they were genotyped on the H3Africa 2.5 million SNP chip. Genotypes were extracted from the Illumina genotype file and converted to Plink format using the H3ABioNet Nextflow topbottom.nf workflow in the H3ABioNet GWAS pipeline (https://github.com/h3abionet/h3agwas).

\subsection{Statistical analysis}

\subsubsection{Differential Gene Expression analysis.}

The $2^{-\Delta \Delta \mathrm{CT}}$ method was applied to determine gene expression levels for each individual (Livak and Schmittgen, 2001) and results used to compare the relative expression of MIF between HAT phenotypes. The Shapiro-Wilk test was initially used to test the null hypothesis $\left(\mathrm{H}_{0}\right)$ that the level of expression followed a normal distribution (p-value > 0.05). As MIF expression levels did not follow a normal distribution, we used the non-parametric Wilcoxon-test to perform the intergroup comparisons. This tested the $\mathrm{H}_{0}$ that there was no difference in the mean expression of $M I F$ between the phenotypes. We also used a paired Wilcoxon-test to compare $M I F$ expression level in the same patient before and after treatment. Association of MIF expression levels with recorded covariates (gender, age, disease focus and HAT phenotypes) were also analysed using univariate and multivariate linear regression models. All data are presented as means $\pm \mathrm{CI}_{95}$ and pairs of conditions and $p$-values $<0.05$ were considered 
significant. All analyses were performed with R 3.4.4 software (R Development Core Team, 2018).

\subsubsection{Expression QTL analysis.}

A genome wide association study was undertaken in Plink to identify SNP associated with MIF expression levels. Quality control of the genotyped data was performed using Plink 1.9. Closely related samples were identified and one of each pair of close relatives were removed. We removed duplicate samples and samples with missing data $>5 \%$ and loci with minor allele frequencies $<5 \%$ and loci which were not in Hardy-Weinberg equilibrium ( $\mathrm{p}<0.001)$. We created a Plink phenotype file (Sample ID, sex, age, expression of MIF) containing 172 samples constituted of 29 controls, 128 cases and 15 individuals with latent infections for whom both expression and genotype data were available. We used linear regression in Plink 1.9 to perform eQTL analysis using the "linear" function, including the disease status as a covariate. Loci where significant eQTL were detected were reanalysed using a much denser set of SNP markers obtained by imputation from our genotype data and 1000 genomes reference data. Imputation was done by H3ABioNet at the University of Capetown using 1000 genome project genotypes as reference (Genomes Project et al., 2015).

\section{Results}

3.1. MIF expression in HAT patients, individuals with latent infections and endemic controls

MIF mRNA quantification was conducted on 212 samples that consisted of healthy endemic controls $n=46(21.70 \%)$ and T. b. gambiense-infected individuals that were subdivided into two phenotypes: 1) patients with active HAT $n=141(66.51 \%)$ of whom 33 (23.40\%) were sampled again after treatment, and 2) individuals with latent infections $n=25$ (11.79\%) who tested positive in serology, but were negative upon microscopic examination and exhibited few or no symptoms. These individuals were followed for a period of at least two years and none developed detectable blood parasitaemia during their follow up despite remaining positive in serology. The sex ratio (male: female) of the study population was 1.41 $(124 / 88)$ and the mean age (range) was 32.76 (5-85) years. Detailed characteristics of the study cohort are provided in Table S1.

MIF expression levels were assayed by RT-qPCR and compared between groups of individuals. As shown in Fig 1 and Table S2, MIF expression levels were found to be significantly elevated in patients $(1.25 \pm 0.07 ; p<0.0001)$ and individuals with a latent infection 
$(1.23 \pm 0.13 ; p=0.0005)$ when compared to controls $(0.94 \pm 0.11)$. No expression differences were observed between HAT cases and individuals with latent infections $(p=0.809)$. As shown in Table 1 the HAT status was the main factor explaining MIF expression levels in a multivariate linear regression model including gender, age and disease focus. These results suggest that MIF expression is induced during $T$. $b$. gambiense infection in humans but that expression levels are not correlated with the clinical status. MIF mean expression levels decreased significantly in patients after treatment $(1.40 \pm 0.18$ before treatment versus $0.99 \pm 0.10$ after treatment; $p=0.0001$ ) and were similar to expression levels observed in controls $(0.94 \pm 0.11 ; p=0.255)$.

\subsection{Localisation of MIF expression quantitative trait loci}

We next performed an eQTL analysis using our MIF expression data and genotype data available for a subset of samples to identify SNP loci that were associated with MIF expression. In all e-QTLs analysis of MIF expression, the infection status was included as a covariate in the regression analysis. Samples were genotyped on the H3Africa Illumina SNP chip that contains 2.5 million SNP loci and is enriched for common African SNPs. We found SNPs at four loci, located on chromosomes 1, 3, 4 and 10 respectively, that had significant associations with $M I F$ expression $\left(\mathrm{p}<10^{-7}\right)($ Fig 2 , Table 2$)$. We did not observe any association signal on chromosome 22 at the MIF locus. SNPs were annotated with the closest gene(s) using Genome Reference Consortium Human Build 37 (GRCh37/hg19) data (Table 3). In particular, the chemokine (C-X-C motif) ligand 13 (CXCL13) gene, involved in both inflammatory and B-cell activation, was found to be proximal to the index SNP on chromosome $4\left(\mathrm{p}=7.12 \times 10^{-8}\right)$. Genetic association at the four identified loci were then further explored by the mean of imputation to increase the number of SNPs in these chromosomal regions (Fig 2b, 2c, 2d, 2e). At each candidate loci, additional SNPs were found to be significantly associated with MIF expression levels thus supporting the genome wide association results (Table S3). A sharp peak of association was observed for the eQTL on chromosome 3 whereas association signals were broader for the eQTLs on chromosome 1, 4 and 10.

In order to assess the importance of $T . \quad b$. gambiense infected individuals in the identification of MIF eQTLs, we repeated the analysis for the four identified regions on chromosomes 1, 3, 4 and 10 on several groups of individuals: all, HAT cases and controls and infected individuals only (HAT cases and latent infections). In all cases lower mean p-values were obtained in the analysis excluding controls (Fig 3) although effectives were lower in this analysis. Most controls had low MIF expression levels and were thus probably not much 
informative in the analysis. This was particularly true for the e-QTLs on chromosomes 3 and

24810 for which the strength of genetic association appears highly dependent of T. b. gambiense infected individuals.

\section{Discussion}

Although several studies have investigated the role of MIF polymorphisms rs36086171 and rs12483859 in HAT resistance/susceptibility (Ahouty et al., 2017; Kabore et al., 2017; Ofon et al., 2017), the present study is the first to look at MIF expression levels in a HAT endemic population containing individuals with latent infections. The principal finding was that, as observed for other infectious diseases, MIF expression is elevated during $T$. $b$. gambiense infection. However, levels did not correlate with disease severity as expression was found to be elevated in both HAT patients with active disease and individuals harbouring latent infections. A second major finding was the identification of four eQTLs involved in the control of MIF expression that may be relevant in wide array of disease settings. This demonstrates that studying humans infected with pathogens that stimulate an immune response may provide new insights into wider immune regulation mechanisms.

MIF is known to be a critical upstream mediator of innate immunity and is involved in a number of pathophysiological inflammatory processes such as glomerulonephritis (Yang et al., 1998) and asthma (Mizue et al., 2005). It is a pleiotropic cytokine produced by a variety of immune cells, including lymphocytes, macrophages and pituitary cells (Calandra et al., 1995). $M I F$ expression is regulated by several factors, such as exposure to endotoxins, inflammatory cytokines and glucocorticoids, and exhibits a wide range of immune activities. This can include the induction of the Toll-like receptor 4 (TLR4) gene and the induction of inflammatory cytokines, such as INFG, TNFA, IL8, IL6 or IL12. MIF is also known to sustain macrophage activation and the secretion of microbicidal molecules, including nitric oxide (Rosado and Rodriguez-Sosa, 2011).

Up-regulation of $M I F$ expression has been reported for many infectious and parasitic diseases, demonstrating that $M I F$ can play either a protective or deleterious role in the immune response to different pathogens (Rosado and Rodriguez-Sosa, 2011). For example, the TLR4mediated response to $T$. cruzi has been reported as one of the main pathways involved in the early production of cytokines associated with human innate immunity during the acute stage (Oliveira et al., 2004). Other studies have shown that MIF secreted by activated macrophages plays an important role in innate immune defences against Mycobacterium tuberculosis by 
acting in an autocrine fashion to inhibit the growth of virulent mycobacteria (Oddo et al., 2005). Low expression alleles of $M I F$ were also shown to confer an increased risk of $M$. tuberculosis disease in some populations (Das et al., 2013). In the present study, we found that MIF expression was significantly upregulated during T. $b$. gambiense infections in both patients with active disease and individuals harbouring latent infections. T. $b$ gambiense thus seems to be a potent inducer of MIF in humans but MIF does not appear to control infection outcome, at least in the Guinean population. This is in contrast to a previous study in mice, where MIF expression was found to be increased in susceptible mice infected by T. brucei. In this model, MIF was proposed to be a major element promoting the most prominent pathological features of experimental trypanosomiasis (Stijlemans et al., 2014). Results presented here suggest that this may not be the case in our Guinean cohort. Interestingly other proinflammatory cytokines that are modulated by MIF, including IL8 and IL6, have been found to be significantly increased in individuals with latent infections (Gineau et al., 2016; Ilboudo et al., 2014). In contrast, susceptibility to disease was associated with increased levels of immunosuppressive molecules, such as IL-10 and soluble HLA-G (Gineau et al., 2016; Ilboudo et al., 2014). This suggest that although MIF seems to be induced during T. b. gambiense infections, control of resistance/susceptibility to HAT occurs downstream of MIF activation in our study population.

This study has also identified four trans-acting eQTLs that are associated with the control of MIF expression. No signs of association where found at the MIF locus on chromosome 22, despite multiple cis-eQTL SNP being reported by the larger study undertaken by GTEx Consortium (GTEx Consortium, 2015). This could be due to the lower coverage of the H3Africa Illumina SNP chip in this region. It is noteworthy however that only suggestive genetic associations were observed between $M I F$ polymorphisms and HAT in this Guinean population (Kabore et al., 2017). Another explanation is that MIF expression regulatory polymorphisms are too rare in this population for associations to be detected with our sample size. Importantly, most studies examining MIF regulation in infectious disease have focused on cis-regulatory elements (Rosado and Rodriguez-Sosa, 2011). Our study suggests that other loci in the genome may also play an important role and should be investigated further to better understand the mechanisms of $M I F$ regulation and implication in disease exacerbation or infection control. The eQTL on chromosome 4 encompasses the CXCL13 gene. Excessive CXCL13 production has been associated with the development of systemic lupus erythematosus nephritis and recombinant CXCL13 has been shown to induce the up-regulation of MIF expression in human podocytes in vitro (Worthmann et al., 2014). Interestingly two 
genes coding for RNA regulatory molecules, a small nucleolar RNA (SNORD2) and a micro

313 RNA (MIR3924), were identified on chromosome 10 in this study, neither of these appeared to have any homology with sequences at the MIF locus suggesting that any regulatory effect that they might have on $M I F$ is indirect through other genes. However, we only considered genes that either encompassed the significant SNP or were the closest gene when the SNP was located in an intergenic region. As shown with our imputed data (Fig 2), association signals can extend up to a $100 \mathrm{~kb}$ on chromosomes 3,4 and 10 suggesting that other candidate genes involved in $M I F$ regulation may be located further away from the index SNPs.

Furthermore, we found that the most significant eQTLs that may be involved in MIF expression regulation were obtained in analyses using infected individuals only (including HAT patients and individuals with latent infections) and excluding controls (Fig 3). This would suggest that $M I F$ is actively regulated by trans-acting genes only when there is an inflammatory response, and that this trans-regulation dominates over any cis-regulation. At other times basal expression may be cis-regulated but our sample of 29 controls may have been too small to detect this or there may be no common regulatory variants at $M I F$ in this population. This suggests that the T. $b$. gambiense infection has boosted the power to detect genetic loci controlling MIF expression. As potent inducers of immune responses, pathogens may thus serve as a valuable tool that may increase the power to unravel regulatory mechanisms involved in the control of critical mediators of immunity in human populations.

\section{Conclusion}

This study has shown that T. b. gambiense infection induces MIF expression in humans and has enabled us to locate four trans-acting eQTLs controlling its expression. As most pathogens are potent inducers of immunological molecules, such as MIF, our study demonstrates that combining clinical phenotypes, whole genome genotypes and expression data in infectious diseases, may contribute to improving our knowledge of immune regulation and susceptibility to other immune-pathophysiological diseases.

\section{Acknowledgments}

339 We thank the subjects who generously donated their specimens and the field workers from the 340 Guinean HAT foci for their dedication in collecting and processing these specimens. We 341 particularly acknowledge the HAT National Control Program of Guinea and all the technicians 342 from the HAT team of the CIRDES (Bobo-Dioulasso, Burkina-Faso) for their help in sampling. 343 We would like to acknowledge also H3ABioNet, funded under grant number U41HG006941, 
for the data provided by their imputation service. This study was funded through the Wellcome

345 Trust (study number 099310/Z/12/Z) awarded to the TrypanoGen Consortium, members of H3Africa (099310). We are grateful to the funders, who had no role in the design and conduct of the study; collection, management, analysis \& interpretation of the data, and preparation, review, or approval of the manuscript.

\section{References}

Ahouty, B., Koffi, M., Ilboudo, H., Simo, G., Matovu, E., Mulindwa, J., Hertz-Fowler, C., Bucheton, B., Sidibé, I., Jamonneau, V., MacLeod, A., Noyes, H., N’Guetta, S.P., 2017. Candidate genes-based investigation of susceptibility to Human African Trypanosomiasis in Côte d'Ivoire. PLoS Negl. Trop. Dis. https://doi.org/10.1371/journal.pntd.0005992

Bozza, F.A., Gomes, R.N., Japiassú, A.M., Soares, M., Castro-Faria-Neto, H.C., Bozza, P.T., Bozza, M.T., 2004. Macrophage migration inhibitory factor levels correlate with fatal outcome in sepsis. Shock. https://doi.org/10.1097/01.shk.0000140305.01641.c8

Buscher, P., Cecchi, G., Jamonneau, V., Priotto, G., 2017. Human African trypanosomiasis. Lancet 390, 2397-2409. https://doi.org/10.1016/S0140-6736(17)31510-6

Calandra, T., 2003. Macrophage migration inhibitory factor and host innate immune responses to microbes. Scand J Infect Dis 35, 573-576.

Calandra, T., Bernhagen, J., Metz, C.N., Spiegel, L.A., Bacher, M., Donnelly, T., Cerami, A., Bucala, R., 1995. MIF as a glucocorticoid-induced modulator of cytokine production. Nature. https://doi.org/10.1093/cje/ber041

Camara, M., Camara, O., Ilboudo, H., Sakande, H., Kaboré, J., N’Dri, L., Jamonneau, V., Bucheton, B., 2010. Sleeping sickness diagnosis: Use of buffy coats improves the sensitivity of the mini anion exchange centrifugation test. Trop. Med. Int. Heal. https://doi.org/10.1111/j.1365-3156.2010.02546.x

Checchi, F., Filipe, J.A.N., Barrett, M.P., Chandramohan, D., 2008. The Natural Progression of Gambiense Sleeping Sickness: What Is the Evidence? PLoS Negl. Trop. Dis. https://doi.org/10.1371/journal.pntd.0000303

Cooper, A., Ilboudo, H., Alibu, V.P., Ravel, S., Enyaru, J., Weir, W., Noyes, H., Capewell, P., Camara, M., Milet, J., Jamonneau, V., Camara, O., Matovu, E., Bucheton, B., MacLeod, A., 2017. APOL1 renal risk variants have contrasting resistance and susceptibility associations with African trypanosomiasis renal risk variants have contrasting resistance and susceptibility associations with African trypanosomiasis. Elife 6. 
https://doi.org/10.7554/eLife.25461

Cutrullis, R.A., Petray, P.B., Schapachnik, E., Sánchez, R., Postan, M., González, M.N., Martín, V., Corral, R.S., 2013. Elevated Serum Levels of Macrophage Migration Inhibitory Factor Are Associated with Progressive Chronic Cardiomyopathy in Patients with Chagas Disease. PLoS One. https://doi.org/10.1371/journal.pone.0057181

Das, R., Koo, M.S., Kim, B.H., Jacob, S.T., Subbian, S., Yao, J., Leng, L., Levy, R., Murchison, C., Burman, W.J., Moore, C.C., Scheld, W.M., David, J.R., Kaplan, G., MacMicking, J.D., Bucala, R., 2013. Macrophage migration inhibitory factor (MIF) is a critical mediator of the innate immune response to Mycobacterium tuberculosis. Proc Natl Acad Sci U S A 110, E2997-3006. https://doi.org/10.1073/pnas.1301128110

Garcia, A., Courtin, D., Solano, P., Koffi, M., Jamonneau, V., 2006. Human African trypanosomiasis: connecting parasite and host genetics. Trends Parasitol 22, 405-409. https://doi.org/10.1016/j.pt.2006.06.011

Garcia, A., Jamonneau, V., Magnus, E., Laveissière, C., Lejon, V., N'Guessan, P., N'Dri, L., Van Meirvenne, N., Büscher, P., 2000. Follow-up of Card Agglutination Trypanosomiasis Test (CATT) positive but apparently aparasitaemic individuals in Cote d'Ivoire: Evidence for a complex and heterogeneous population. Trop. Med. Int. Heal. https://doi.org/10.1046/j.1365-3156.2000.00623.x

Genomes Project, C., Auton, A., Brooks, L.D., Durbin, R.M., Garrison, E.P., Kang, H.M., Korbel, J.O., Marchini, J.L., McCarthy, S., McVean, G.A., Abecasis, G.R., 2015. A global reference for human genetic variation. Nature 526, 68-74. https://doi.org/10.1038/nature15393

Gineau, L., Courtin, D., Camara, M., Ilboudo, H., Jamonneau, V., Dias, F.C., Tokplonou, L., Milet, J., Mendonça, P.B., Castelli, E.C., Camara, O., Camara, M., Favier, B., RouasFreiss, N., Moreau, P., Donadi, E.A., Bucheton, B., Sabbagh, A., Garcia, A., 2016. Human Leukocyte Antigen-G: A Promising Prognostic Marker of Disease Progression to Improve the Control of Human African Trypanosomiasis. Clin. Infect. Dis. https://doi.org/10.1093/cid/ciw505

GTEx Consortium, 2015. Human genomics. The Genotype-Tissue Expression (GTEx) pilot analysis: multitissue gene regulation in humans. Science (80-. ). https://doi.org/10.1126/science.1262110

Ilboudo, H., Bras-Goncalves, R., Camara, M., Flori, L., Camara, O., Sakande, H., Leno, M., Petitdidier, E., Jamonneau, V., Bucheton, B., 2014. Unravelling human trypanotolerance: IL8 is associated with infection control whereas IL10 and TNFalpha are associated with 
subsequent disease development. PLoS Pathog 10, e1004469.

https://doi.org/10.1371/journal.ppat.1004469

Ilboudo, H., Jamonneau, V., Camara, M., Camara, O., Dama, E., Léno, M., Ouendeno, F., Courtin, F., Sakande, H., Sanon, R., Kaboré, J., Coulibaly, B., N’Dri, L., Diarra, A., N'Goran, E., Bucheton, B., 2011. Diversity of response to Trypanosoma brucei gambiense infections in the Forecariah mangrove focus (Guinea): Perspectives for a better control of sleeping sickness. Microbes Infect. https://doi.org/10.1016/j.micinf.2011.05.007

Ilboudo, H., Noyes, H., Mulindwa, J., Kimuda, M.P., Koffi, M., Kaboré, J.W., Ahouty, B., Ngoyi, D.M., Fataki, O., Simo, G., Ofon, E., Enyaru, J., Chisi, J., Kamoto, K., Simuunza, M., Alibu, V.P., Lejon, V., Jamonneau, V., Macleod, A., Camara, M., Bucheton, B., Hertz-Fowler, C., Sidibe, I., Matovu, E., 2017. Introducing the TrypanoGEN biobank: A valuable resource for the elimination of human African trypanosomiasis, in: PLoS Neglected Tropical Diseases. https://doi.org/10.1371/journal.pntd.0005438

Jamonneau, V., Bucheton, B., Kaboré, J., Ilboudo, H., Camara, O., Courtin, F., Solano, P., Kaba, D., Kambire, R., Lingue, K., Camara, M., Baelmans, R., Lejon, V., Buscher, P., 2010. Revisiting the immune trypanolysis test to optimise epidemiological surveillance and control of sleeping sickness in West Africa. PLoS Negl. Trop. Dis. https://doi.org/10.1371/journal.pntd.0000917

Jamonneau, V., Ilboudo, H., Kaboré, J., Kaba, D., Koffi, M., Solano, P., Garcia, A., Courtin, D., Laveissière, C., Lingue, K., Büscher, P., Bucheton, B., 2012. Untreated Human Infections by Trypanosoma brucei gambiense are Not 100\% Fatal. PLoS Negl Trop Dis. https://doi.org/10.1371/journal.pntd.0001691

Kabore, J.W., Ilboudo, H., Noyes, H., Camara, O., Kabore, J., Camara, M., Koffi, M., Lejon, V., Jamonneau, V., MacLeod, A., Hertz-Fowler, C., Belem, A.M.G., Matovu, E., Bucheton, B., Sidibe, I., 2017. Candidate gene polymorphisms study between human African trypanosomiasis clinical phenotypes in Guinea. PLoS Negl. Trop. Dis. https://doi.org/10.1371/journal.pntd.0005833

Kimura, K., Nagaki, M., Nishihira, J., Satake, S., Kuwata, K., Moriwaki, H., 2006. Role of macrophage migration inhibitory factor in hepatitis B virus-specific cytotoxic-Tlymphocyte-induced liver injury. Clin. Vaccine Immunol. https://doi.org/10.1128/CVI.13.3.415-419.2006

Ledderose, C., Heyn, J., Limbeck, E., Kreth, S., 2011. Selection of reliable reference genes 
for quantitative real-time PCR in human T cells and neutrophils. BMC Res. Notes. https://doi.org/10.1186/1756-0500-4-427

Livak, K.J., Schmittgen, T.D., 2001. Analysis of relative gene expression data using real-time quantitative PCR and the 2(-Delta Delta C(T)) Method. Methods. https://doi.org/10.1006/meth.2001.1262

Magnus, E., Vervoort, T., Van Meirvenne, N., 1978. A card-agglutination test with stained trypanosomes (CATT) for the serological diagnosis of T. b. gambiense trypanosomiasis. Ann. Soc. Belg. Med. Trop. (1920).

McDevitt, M.A., Xie, J., Shanmugasundaram, G., Griffith, J., Liu, A., McDonald, C., Thuma, P., Gordeuk, V.R., Metz, C.N., Mitchell, R., Keefer, J., David, J., Leng, L., Bucala, R., 2006. A critical role for the host mediator macrophage migration inhibitory factor in the pathogenesis of malarial anemia. J. Exp. Med. https://doi.org/10.1084/jem.20052398

Mizue, Y., Ghani, S., Leng, L., McDonald, C., Kong, P., Baugh, J., Lane, S.J., Craft, J., Nishihira, J., Donnelly, S.C., Zhu, Z., Bucala, R., 2005. Role for macrophage migration inhibitory factor in asthma. Proc. Natl. Acad. Sci. https://doi.org/10.1073/pnas.0507189102

Morand, E.F., Leech, M., Weedon, H., Metz, C., Bucala, R., Smith, M.D., 2002. Macrophage migration inhibitory factor in rheumatoid arthritis: clinical correlations. Rheumatology (Oxford). https://doi.org/10.1093/rheumatology/41.5.558

Oddo, M., Calandra, T., Bucala, R., Meylan, P.R., 2005. Macrophage migration inhibitory factor reduces the growth of virulent Mycobacterium tuberculosis in human macrophages. Infect Immun 73, 3783-3786. https://doi.org/10.1128/IAI.73.6.37833786.2005

Ofon, E., Noyes, H., Mulindwa, J., Ilboudo, H., Simuunza, M., Ebo’o, V., Njiokou, F., Koffi, M., Bucheton, B., Fogue, P., Hertz-Fowler, C., MacLeod, A., Simo, G., 2017. A polymorphism in the haptoglobin, haptoglobin related protein locus is associated with risk of human sleeping sickness within Cameroonian populations. PLoS Negl. Trop. Dis. https://doi.org/10.1371/journal.pntd.0005979

Oliveira, A.-C., Peixoto, J.R., de Arruda, L.B., Campos, M.A., Gazzinelli, R.T., Golenbock, D.T., Akira, S., Previato, J.O., Mendonca-Previato, L., Nobrega, A., Bellio, M., 2004. Expression of Functional TLR4 Confers Proinflammatory Responsiveness to Trypanosoma cruzi Glycoinositolphospholipids and Higher Resistance to Infection with T. cruzi. J. Immunol. https://doi.org/10.4049/jimmunol.173.9.5688 R Development Core Team, 2018. R statistical software. R A Lang. Environ. Stat. Comput. 
https://doi.org/10.1016/j.marpetgeo.2016.04.009

480

481

482

483

484

485

486

487

488

489

490

491

492

493

494

495

496

497

498

499

500

501

502

503

504

505

506

507

508

509

510

511

512

Regis, E.G., Barreto-de-Souza, V., Morgado, M.G., Bozza, M.T., Leng, L., Bucala, R., BouHabib, D.C., 2010. Elevated levels of macrophage migration inhibitory factor (MIF) in the plasma of HIV-1-infected patients and in HIV-1-infected cell cultures: A relevant role on viral replication. Virology. https://doi.org/10.1016/j.virol.2009.12.018

Renner, P., Roger, T., Calandra, T., P., R., T., R., 2005. Macrophage migration inhibitory factor: Gene polymorphisms and susceptibility to inflammatory diseases. Clin. Infect. Dis. https://doi.org/10.1086/432009

Reyes, J.L., Terrazas, L.I., Espinoza, B., Cruz-Robles, D., Soto, V., Rivera-Montoya, I., Gómez-García, L., Snider, H., Satoskar, A.R., Rodríguez-Sosa, M., 2006. Macrophage migration inhibitory factor contributes to host defense against acute Trypanosoma cruzi Infection. Infect. Immun. https://doi.org/10.1128/IAI.01648-05

Rosado, J. de D., Rodriguez-Sosa, M., 2011. Macrophage migration inhibitory factor (MIF): A Key player in protozoan infections. Int. J. Biol. Sci. https://doi.org/10.7150/ijbs.7.1239

Simarro, P.P., Cecchi, G., Paone, M., Franco, J.R., Diarra, A., Ruiz, J.A., Fevre, E.M., Courtin, F., Mattioli, R.C., Jannin, J.G., 2010. The Atlas of human African trypanosomiasis: a contribution to global mapping of neglected tropical diseases. Int $\mathbf{J}$ Heal. Geogr 9, 57. https://doi.org/10.1186/1476-072X-9-57

Stijlemans, B., Leng, L., Brys, L., Sparkes, A., Vansintjan, L., Caljon, G., Raes, G., Van Den Abbeele, J., Van Ginderachter, J.A., Beschin, A., Bucala, R., De Baetselier, P., 2014. MIF contributes to Trypanosoma brucei associated immunopathogenicity development. PLoS Pathog 10, e1004414. https://doi.org/10.1371/journal.ppat.1004414

Truc, P., Formenty, P., Diallo, P.B., Komoin-Oka, C., Lauginie, F., 1997. Confirmation of two distinct classes of zymodemes of Trypanosoma brucei infecting man and wild mammals in Cote d'Ivoire: Suspected difference in pathogenicity. Ann. Trop. Med. Parasitol. https://doi.org/10.1080/00034989760356

Vanhamme, L., Paturiaux-Hanocq, F., Poelvoorde, P., Nolan, D.P., Lins, L., Van Den Abbeele, J., Pays, A., Tebabi, P., Van Xong, H., Jacquet, A., Moguilevsky, N., Dieu, M., Kane, J.P., De Baetselier, P., Brasseur, R., Pays, E., 2003. Apolipoprotein L-I is the trypanosome lytic factor of human serum. Nature 422, 83-87. https://doi.org/10.1038/nature01461

Worthmann, K., Gueler, F., Von Vietinghoff, S., Davalos-Mi, A., Wiehler, F., Davidson, A., Witte, T., Haller, H., Schiffer, M., Falk, C.S., Schiffer, L., 2014. Pathogenetic role of glomerular CXCL13 expression in lupus nephritis. Clin. Exp. Immunol. 
Yang, N., Nikolic-Paterson, D.J., Ng, Y.Y., Mu, W., Metz, C., Bacher, M., Meinhardt, a, Bucala, R., Atkins, R.C., Lan, H.Y., 1998. Reversal of established rat crescentic glomerulonephritis by blockade of macrophage migration inhibitory factor (MIF): potential role of MIF in regulating glucocorticoid production. Mol. Med.

Zernecke, A., Bernhagen, J., Weber, C., 2008. Macrophage migration inhibitory factor in cardiovascular disease. Circulation. https://doi.org/10.1161/CIRCULATIONAHA.107.729125

524

525 526

\section{Figure legends}

Fig 1: MIF expression in HAT patients, individuals carrying latent infections and endemic controls. (A) MIF expression levels according to the clinical status: endemic controls $(n=46)$, HAT patients or cases $(n=141)$, latent infections $(n=25)$. (B) MIF expression levels in cases before treatment $(n=33)$ and the same cases after treatment $(n=33)$. 1_Cases BeT: cases before treatment, 2_Cases AfT: cases after treatment, $* * * * p<0.0001, * * \mathrm{p}<0.01$, ns: not significant.

Fig 2: Manhattan plots showing the locations of MIF expression eQTL. The e-QTL analysis was performed by linear regression using the clinical status (HAT patient, latent infection, endemic control) as a covariate. A. eQTL mapping using the whole genome genotype dataset from the Illumina 2.5M SNP chip. Association results after including imputed SNPs at the candidate e-QTLs on chromosome 1 (B.), chromosome 3 (C.), chromosome 4 (D.) and chromosome 10 (E.).

Fig 3: Bar plot representing the mean p-value of significant imputed SNPs at each eQTL locus according to the infection status. For each model the phenotype was used as covariate in the linear regression analysis. Covariate coding: 1-Infected vs Controls $(\mathbf{n = 1 7 2})$ : Controls were coded 1 and both active and latent infections were coded 2; 2- HAT cases versus Controls (n=157): Controls and active cases were coded 1 and 2 and latent infections were coded 0 (missing) and were excluded; 3-HAT cases versus Latent $(\mathbf{n = 1 4 3})$ : Latent infections were coded 1 , active cases were coded 2, controls were coded 0 and were excluded. 
Table 1: Analysis of $M I F$ expression levels according to recorded covariates

\begin{tabular}{llccc}
\hline Covariates & \multicolumn{3}{c}{ Univariate } & Multivariate \\
\cline { 2 - 5 } & $n$ & $M I F^{a}$ & $p$ \\
\hline Sex & 88 & $1.22 \pm 0.10$ & & \\
Females & 124 & $1.16 \pm 0.07$ & 0.3121 & 0.2250 \\
Males & & & & \\
Age & 68 & $1.15 \pm 0.10$ & & 0.2614 \\
$\leq 24$ years & 77 & $1.21 \pm 0.11$ & 0.2866 & \\
$25-39$ years & 67 & $1.19 \pm 0.09$ & & \\
$\geq 40$ years & & & & \\
Disease focus & 61 & $1.22 \pm 0.13$ & & \\
Forecariah & 106 & $1.20 \pm 0.71$ & 0.0951 & \\
Dubreka & 45 & $1.27 \pm 0.13$ & & \\
Boffa & & & & \\
HAT phenotypes & 46 & $0.94 \pm 0.11$ & $<0.0001$ & \\
Controls & 25 & $1.23 \pm 0.13$ & & \\
Latent infections & 141 & $1.25 \pm 0.07$ & & \\
HAT & & & & \\
\hline
\end{tabular}

$543 \quad{ }^{a}$ Mean fold change \pm mean confidence interval

544 Table 2: SNPs reaching genome wide significance in the MIF e-QTL analysis.

\begin{tabular}{l|llllllll}
\hline CHR & SNP & BP & Al & TEST & NMISS & BETA & STAT & $P$ \\
\hline 1 & kgp15347739 & 116918691 & G & ADD & 120 & 0.5036 & 5.937 & $3.04 \mathrm{E}-08$ \\
3 & snp-known116300863 & 115325154 & A & ADD & 149 & 0.4603 & 5.642 & $8.44 \mathrm{E}-08$ \\
4 & kgp20876446 & 78506066 & G & ADD & 120 & 0.5356 & 5.754 & $7.12 \mathrm{E}-08$ \\
10 & snp-known78014716 & 58884536 & C & ADD & 120 & 0.5346 & 5.696 & $9.29 \mathrm{E}-08$ \\
\hline
\end{tabular}

545 CHR: Chromosomes, SNP: single nucleotide polymorphism, A1: minor allele name, BP, base-pair physical

546 position, ADD: additive model. The e-QTL analysis was performed on the whole data set (n=172) using a linear

547 regression model with the clinical status as a covariate.

548 Table 3: Proximal e-QTLs candidate genes controlling MIF expression.

\begin{tabular}{r|llll}
\hline \multicolumn{1}{c}{ CHR } & SNP & Gene name & Gene stable ID & Gene description \\
\hline 1 & kgp15347739 & AL136376.1 & ENSG00000269279.1 & Uncharacterized protein \\
3 & snp-known116300863 & RP11-190P13.1 & ENSG00000244157.1 & $\begin{array}{l}\text { eukaryotic translation initiation factor 4E } \\
\text { family member 2 pseudogene 2 }\end{array}$ \\
3 & snp-known116300863 & GAP43 & ENSG00000172020.8 & growth associated protein 43 \\
4 & kgp20876446 & CXCL13 & ENSG00000156234.7 & chemokine (C-X-C motif) ligand 13 \\
10 & snp-known78014716 & SNORD2 & ENSG00000238707.1 & Small nucleolar RNA SNORD2 \\
10 & snp-known78014716 & MIR3924 & ENSG00000264747.1 & microRNA 3924 \\
\hline
\end{tabular}


549 CHR: Chromosomes, SNP: single nucleotide polymorphism. Each SNP reaching genome wide association 550 significance was annotated with either the gene they were located in or the genes immediately upstream or 551 downstream for intergenic SNPs.

\section{Supporting Information Legends}

553 Table S1: The age and gender characteristics of the different study phenotype groups

554 Table S2: MIF Expression levels according to the HAT clinical status

555 Table S3: SNPs reaching genome wide significance in the MIF e-QTL analysis with 556 imputed data. 


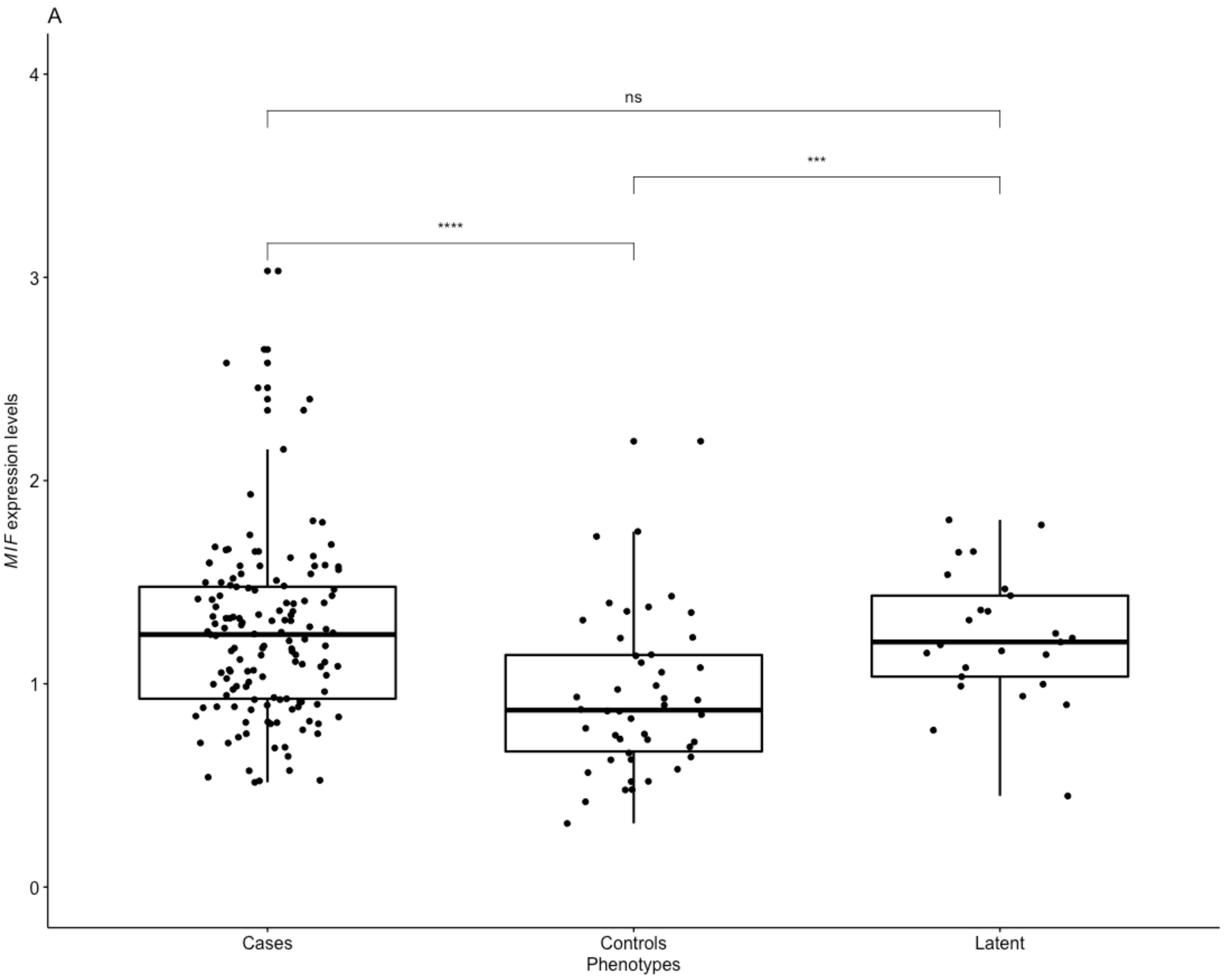


Wilcoxon, $p=0.00011$

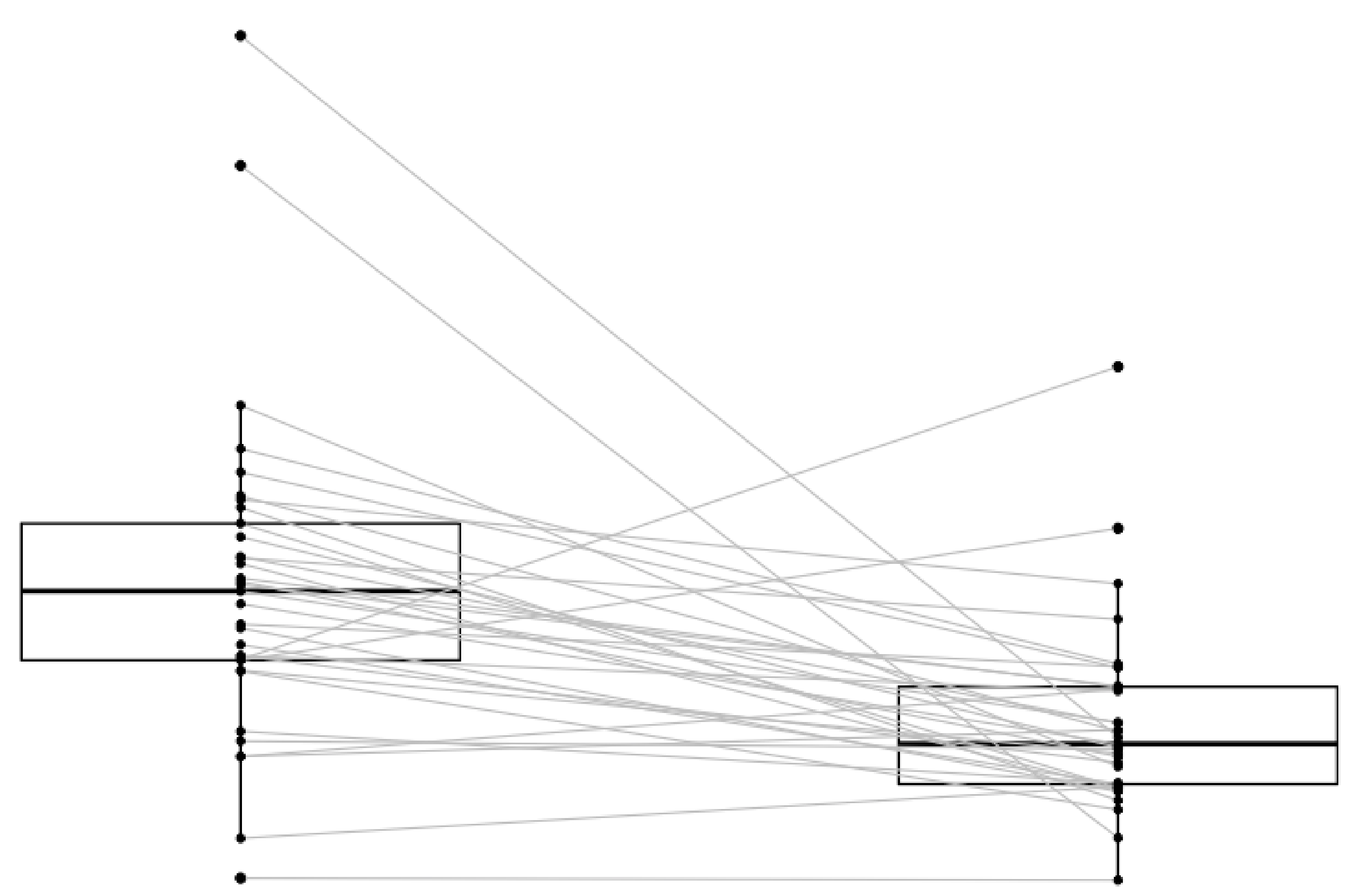




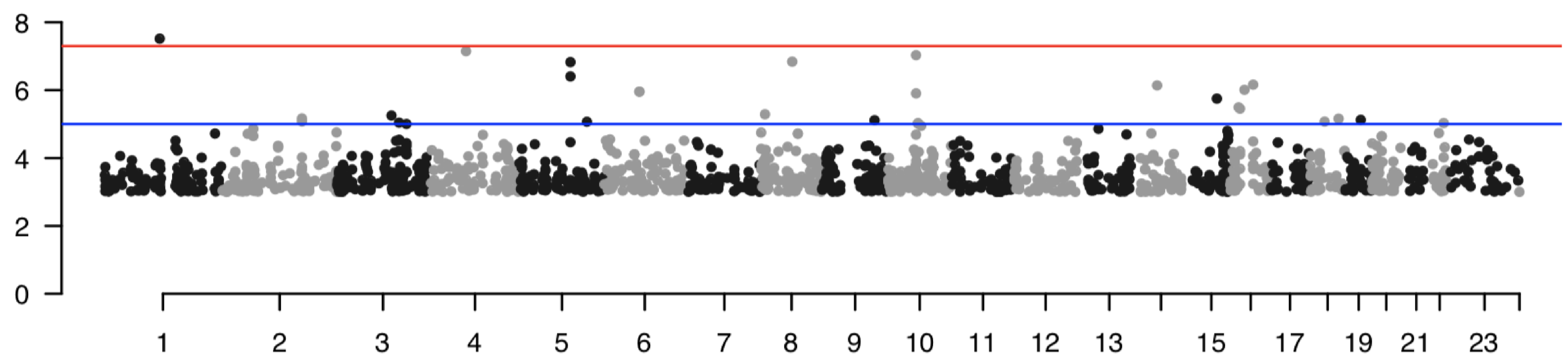

Chromosome

C

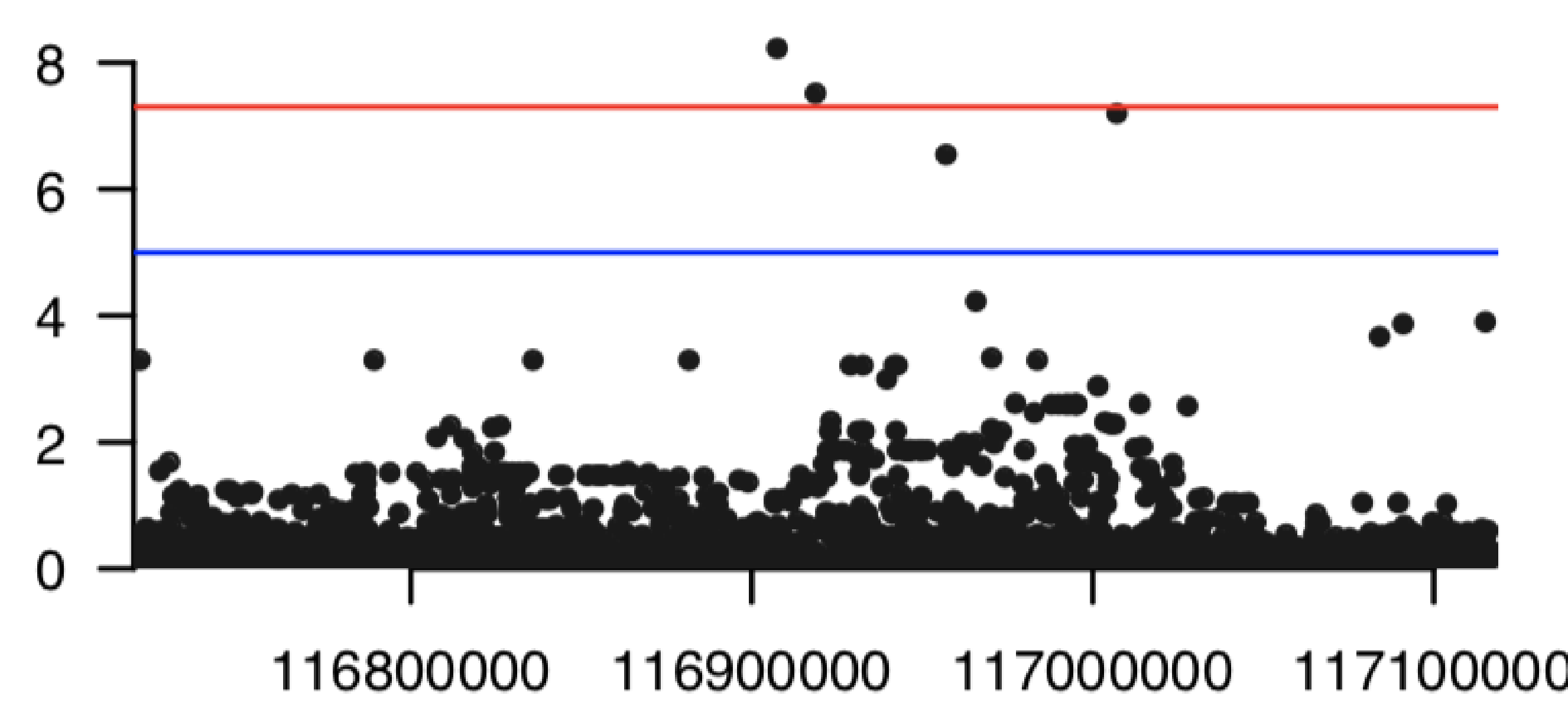

Chromosome 1 position

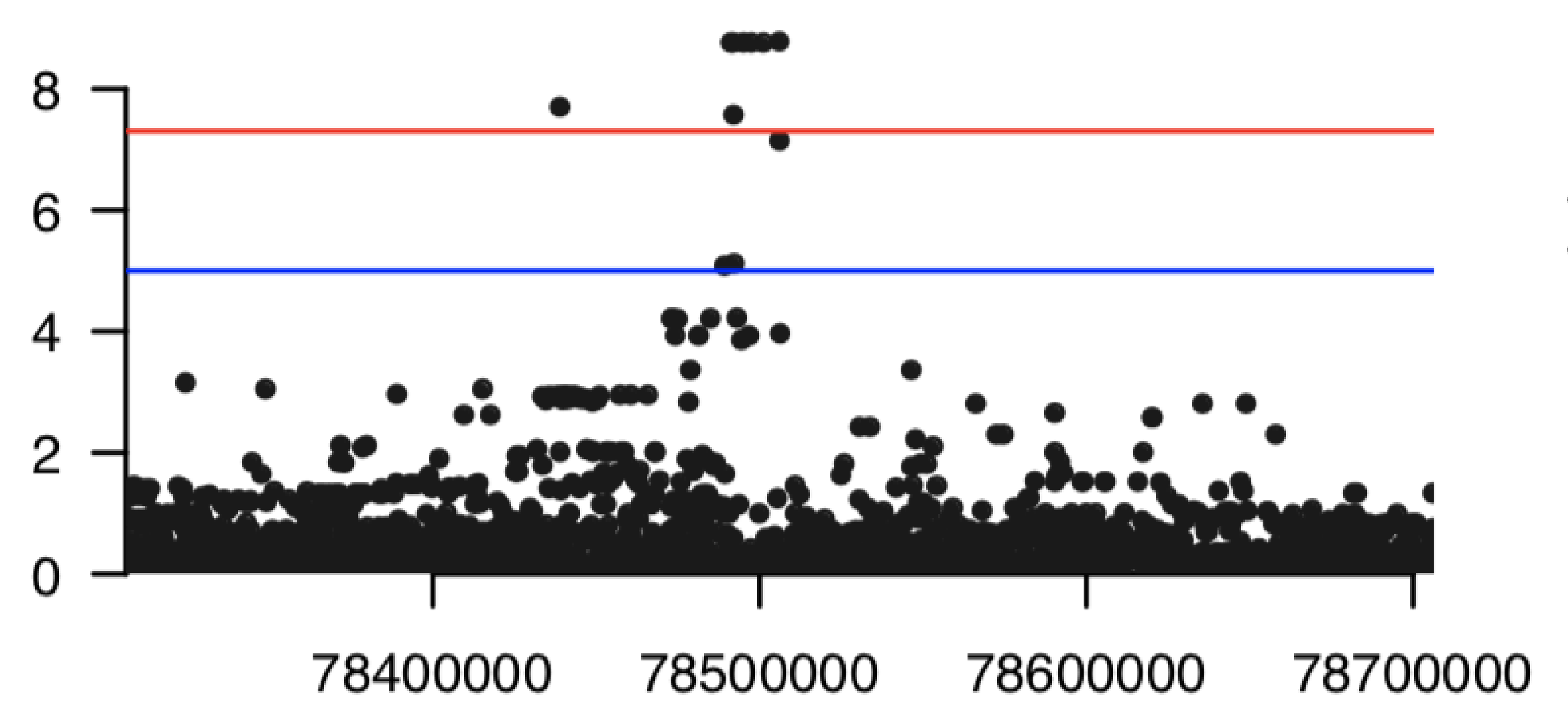

Chromosome 4 position

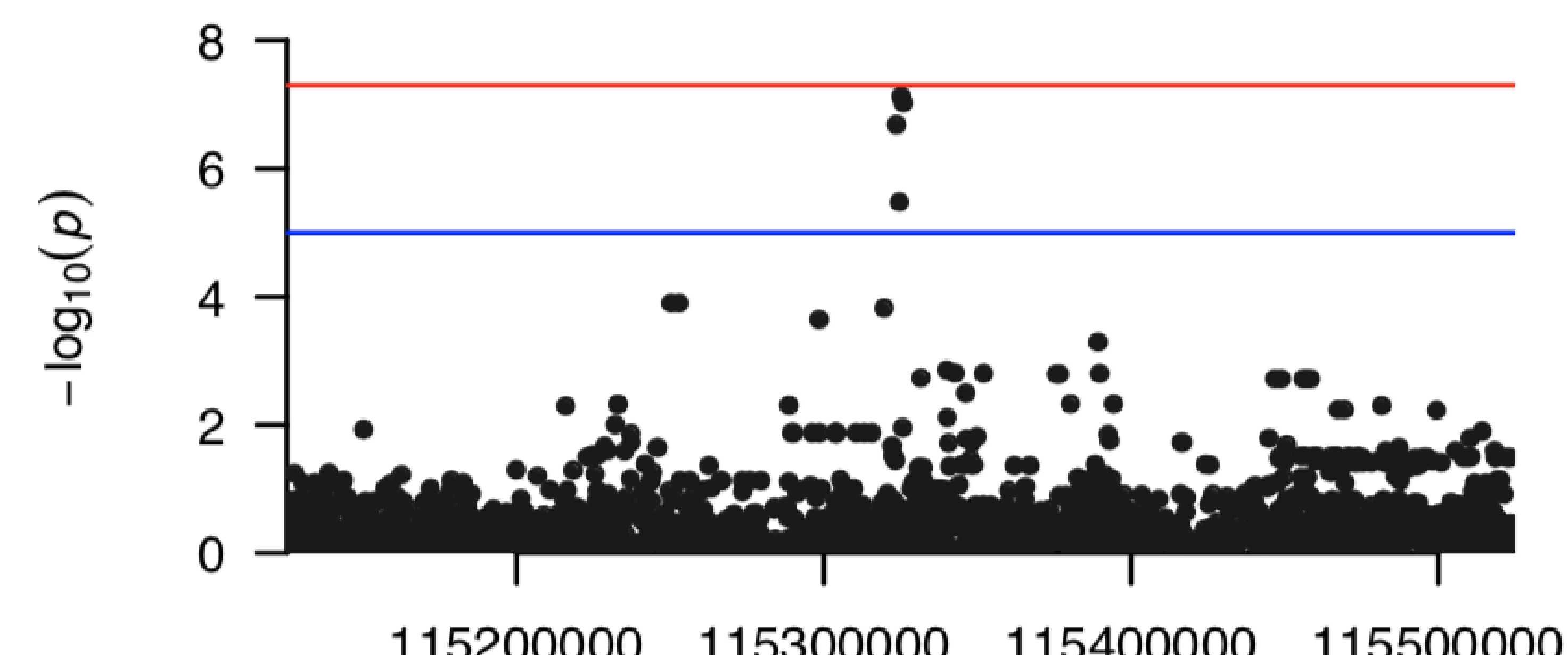

Chromosome 3 position

E

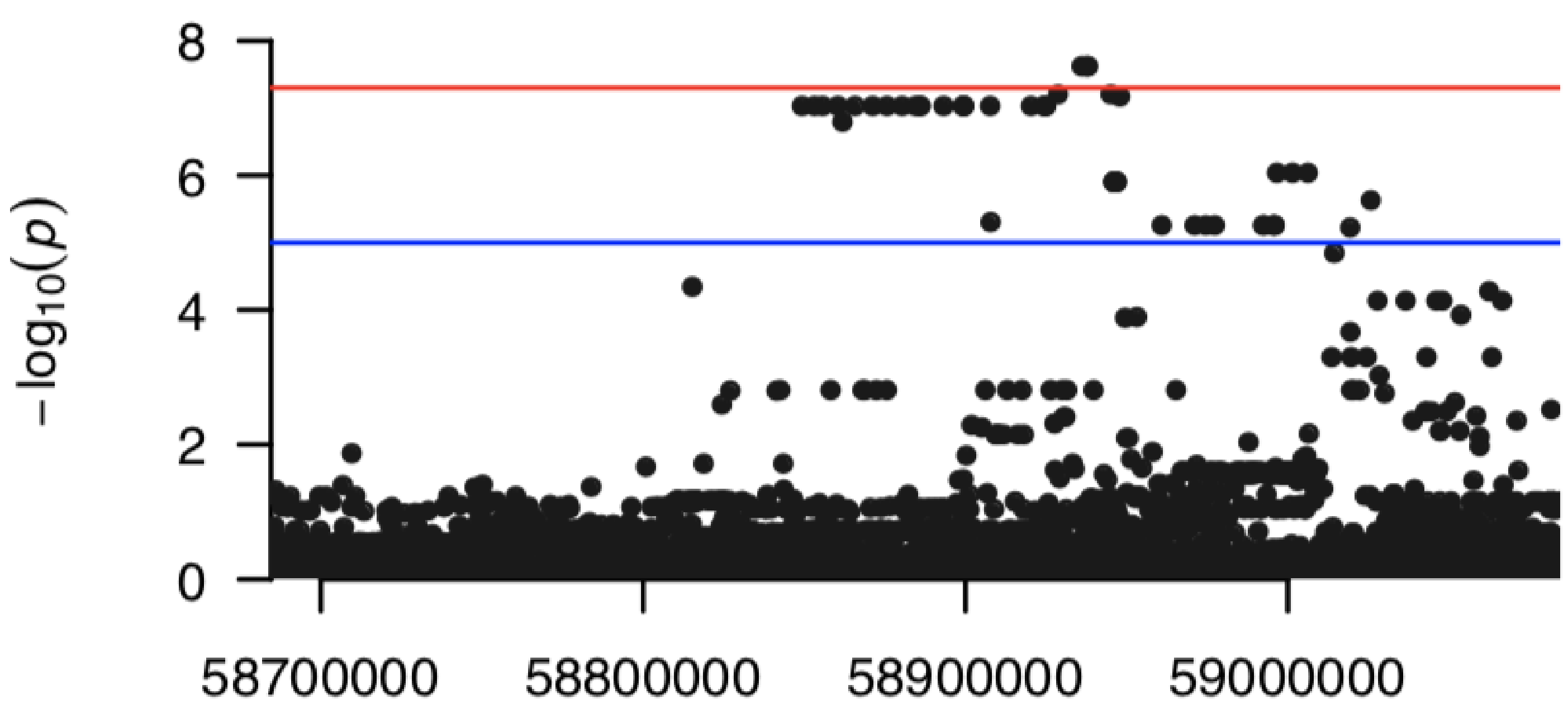

Chromosome 10 position 


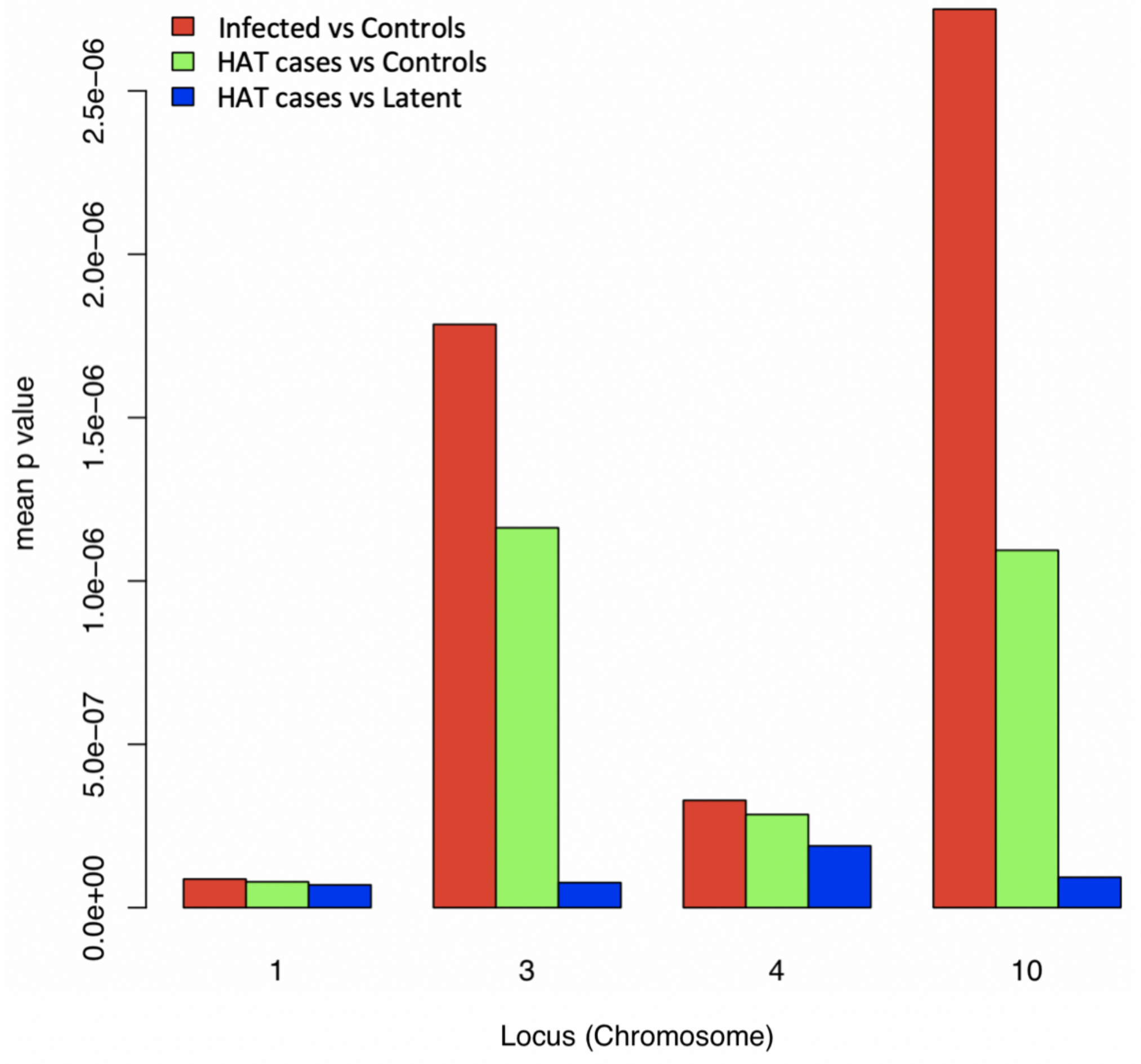

